\title{
Observer-based Diagnosis in Chemical Reaction Networks
}

\author{
Lốrinc Márton $^{1}$ and Gábor Szederkényi ${ }^{2}$ and Katalin M. Hangos ${ }^{2}$
}

\begin{abstract}
This paper proposes a method to diagnose unexpected changes in the dynamic behavior of Chemical Reaction Network models. It is considered that the disturbances can induce changes in the reaction rate coefficients of chemical reactions. Conditions for the estimation of such disturbances are formulated. Using the algebraic properties of kinetic models, on-line observers are designed to monitor the disturbancegenerated modifications in the reaction rate coefficients. An extended disturbance observer is also introduced for such cases when not all the states of the Chemical Reaction Network are measurable. The applicability of the developed method is shown through simulation studies.
\end{abstract}

Keywords: Disturbance Estimation, Nonlinear Observer, Chemical Reaction Network, Lyapunov design

\section{INTRODUCTION}

The design of observer-based diagnosis methods for general nonlinear systems is a challenging task that presents both theoretical and computational difficulties [1]. Therefore, the research efforts in this area are directed toward developing special computationally efficient methods that utilize the specialties of the application field.

Several kinds of important dynamical phenomena in nature or technology can be modeled in the framework of nonnegative systems having the property that the nonnegative orthant is invariant for the dynamics. Notable examples are (bio)chemical kinetic processes, models of disease and population dynamics, a wide range of models in the process industries, and certain economic or transportation processes (see e.g. [2] or [3]).

Chemical Reaction Networks (abbreviated as CRNs) form an important class within the family of smooth nonnegative nonlinear systems with increasing research interest in the last decade [4], because they are suitable for the modeling of complex nonlinear dynamical behavior, but have a mathematically simple and therefore computationally appealing structure. In addition, there are numerous strong results in the literature on the relation between the graph structure and important dynamical properties of CRNs. The first results on the dynamical and other properties of CRNs have appeared in the late 1970's by [5], but they have become widely known in the systems and control literature in the 2000s [6]. Utilizing the strong results on the structural (i.e. parameter

\footnotetext{
*This work was supported by the National Research, Development and Innovation Office of Hungary through grants K115694. The research work of L. Márton was also supported by the Domus Hungarica Scholarship of the Hungarian Academy of Sciences.

${ }^{1}$ Dept. of Electrical Engineering, Sapientia Hungarian University of Transylvania, $540485 \mathrm{Tg}$. Mures, Romania martonl@ms. sapientia.ro

${ }^{2}$ Systems and Control Laboratory, Institute for Computer Science and Control HAS, 1111 Budapest, Hungary hangosescl.sztaki.hu, szederasztaki. hu
}

independent) stability of CRNs, computationally efficient state estimation [7], stabilizing feedback controller design [8] and observer design [9] methods have been developed for this nonlinear system class. These results have paved the way to develop observer-based diagnosis methods for CRNs, too.

For efficient analysis and control, the modelling and identification of CRNs are required. Off-line identification methods for chemical process models were developed e.g. in [10] and [11]. In the paper [12] conditions were formulated for the identifiability of CRNs. Similar identifiability results were developed for system biology models in the study [13].

Disturbance- and state estimation problems for chemical processes, to which CRNs also belong, stayed constantly in focus of the researchers in the last decades due to their importance in industrial production [14]. The parameter- or input disturbances not necessarily lead to malfunction of the process control systems but they could affect both the steady state and transient control performances, and consequently the quality of the production. It is why efficient disturbance estimation methods are necessary for the monitoring and feed-forward disturbance compensation in process systems. Recent approaches for the state- and disturbance estimation in process systems can be found e.g. in [15] in which a robust extended Kalman filter was proposed for simultaneous state and parameter/disturbance estimation in process systems. In the study [16] the virtual sensor approach was applied to estimate unknown disturbances.

The aim of this study is the design of an on-line chemical reaction rate coefficient- and input disturbance estimator for CRNs. First, a reformulated CRN model is proposed that is suitable for on-line estimation of reaction rate changes in these systems. Second, a disturbance observer algorithm and sufficient conditions are formulated for exact on-line estimation of the reaction rate changes in CRNs. The proposed observer design approach exploits the properties of the CRN models. The algorithm was extended for open CRNs with possible input disturbances. Third, a modified disturbance observer structure was proposed for such case when not all the concentration states of the CRN are known during the estimation process.

The rest of the paper is organized as follows: in section II a modeling approach for CRNs with disturbances is presented that facilitates the observer design. Section III introduces the proposed disturbance observers for CRNs. Simulation results are given in section IV. Finally, section V concludes this study. 


\section{CRN MODELS FOR OBSERVER DESIGN}

The dynamic model of CRNs is described below illustrated with a simple example. Then the considered disturbances and their appearance in this model are briefly outlined.

\section{A. Dynamic model of Chemical Reaction Networks}

The dynamic model of a CRN is built upon the following elements [5]:

- Species: $\mathcal{S}:=\left\{S_{1} \ldots S_{n}\right\}$ are constituent molecules undergoing (a series of) chemical reactions.

- Complexes: $\mathcal{C}:=\left\{\mathcal{C}_{1} \ldots \mathcal{C}_{m}\right\}$ are formally linear combinations of the species with integer coefficients, i.e. $\mathcal{C}_{k}:=\sum_{i=1}^{n} \alpha_{k, i} S_{i}$, where $\alpha_{k, i}$ are the stoichiometric coefficients. If $S_{i}$ is not present in $\mathcal{C}_{k}$, then $\alpha_{k, i}=0$.

- Reactions: $\mathcal{R}:=\left\{\mathcal{R}_{1} \ldots \mathcal{R}_{r}\right\}$ where $\mathcal{R}_{k}: \mathcal{C}_{i} \rightarrow \mathcal{C}_{j}$. Here $\mathcal{C}_{i}$ is the reactant (or source) complex, and $\mathcal{C}_{j}$ is the product complex for $k=1, \ldots, r$.

- Reaction rate coefficient: $\kappa_{k}>0$ that is associated to $\mathcal{R}_{k}$ for $k=1, \ldots, r$.

We associate vectors $\mathbf{y}_{k} \in \mathbb{R}^{n}$ to the complexes $\mathcal{C}_{k}$ composed of their stoichiometric coefficients $\alpha_{k i}$ such that $\mathbf{y}_{k, i}=\alpha_{k i}$ for $k=1, \ldots m$. Let us denote by $\mathbf{y}_{k R} \in \mathbb{R}^{n}$ the so-called complex vector associated to a reactant complex, and by $\mathbf{y}_{k P} \in \mathbb{R}^{n}$ the vector associated to the product complex of the $k$ th reaction, i.e. $\mathcal{R}_{k}: \mathcal{C}_{k R} \rightarrow \mathcal{C}_{k P}$.

The reaction vector for the $k$ th reaction is defined as $\mathbf{y}_{k P}-$ $\mathbf{y}_{k R}$. The stoichiometric matrix $N \in \mathbb{R}^{n \times r}$ contains all the reaction vectors of a CRN in its columns.

The CRN model describes the dynamics of the species' concentrations. Let us denote the concentration vector by $\mathbf{c}=\left(\begin{array}{llll}c_{1} & c_{2} & \ldots & c_{n}\end{array}\right) \in \mathbb{R}_{+}^{n}$.

Mass action law: The simplest polynomial rate function corresponds to the so called mass action law, when the reaction rate of the $k$ th reaction $\mathcal{R}_{k}: \sum_{i=1}^{n S} \alpha_{k R, i} S_{k i} \rightarrow \mathcal{C}_{k P}$ is in the following form

$$
r_{k}(\mathbf{c}, \mathbf{k})=\kappa_{k} \prod_{i=1}^{n} c_{i}^{\alpha_{k R, i}}
$$

where $0^{0}:=1$ and $\mathbf{k}=\left(\begin{array}{llll}\kappa_{1} & \kappa_{2} \ldots \kappa_{r}\end{array}\right) \in \mathbb{R}_{+}^{r}$. Let us introduce the monomial vector

$$
\mathbf{p}(\mathbf{c})=\left(p_{1}(\mathbf{c}) p_{2}(\mathbf{c}) \ldots p_{r}(\mathbf{c})\right) \in \mathbb{R}_{+}^{r}
$$

with $p_{k}(\mathbf{c})=\prod_{i=1}^{n} c_{i}^{\alpha_{k R, i}}$ Now we can form the reaction rate vector $\mathbf{r}(\mathbf{c}, \mathbf{k})=\left(r_{1} \ldots r_{r}\right)^{T}$ in the form

$$
\mathbf{r}(\mathbf{c}, \mathbf{k})=\operatorname{diag}(\mathbf{k}) \mathbf{p}(\mathbf{c})=\operatorname{diag}(\mathbf{p}(\mathbf{c})) \mathbf{k}
$$

With these notations the ODE (Ordinary Differential Equation) model of a CRN reads as [17]:

$$
\dot{\mathbf{c}}=N \mathbf{r}(\mathbf{c}, \mathbf{k}), \quad \mathbf{c}(0)=\mathbf{c}_{\circ} .
$$

Example 1: (Plain Edelstein Network)

This model was originally published in [18] for illustrating the phenomena of multiple steady states and hysteresis for a simple biologically motivated reaction network structure. The Edelstein CRN is composed of three species $\left(S_{1}, S_{2}\right.$ and $S_{3}$ ) and six chemical reactions. The reactions describe autocatalytic production and the enzymatic degradation of the species $S_{1}$ [19]. The reaction structure of this CRN has the form:

$$
\begin{array}{r}
S_{1} \underset{\kappa_{2}}{\stackrel{\kappa_{1}}{\rightleftharpoons}} 2 S_{1} \\
S_{1}+S_{2} \underset{\kappa_{4}}{\stackrel{\kappa_{3}}{\rightleftharpoons}} S_{3} \underset{\kappa_{6}}{\stackrel{\kappa_{5}}{\rightleftharpoons}} S_{2}
\end{array}
$$

Let the matrices $N_{R}$ and $N_{P}$ whose columns are the reactant- and product vectors $\left(\mathbf{y}_{k R}\right.$ and $\left.\mathbf{y}_{k P}\right)$. In the case of the Edelstein network they have the form:

$$
N_{R}=N_{P}=\left(\begin{array}{ccccc}
1 & 2 & 1 & 0 & 0 \\
0 & 0 & 1 & 0 & 1 \\
0 & 0 & 0 & 1 & 0
\end{array}\right)
$$

The stoichiometric matrix of the Edelstein network reads as:

$$
N=\left(\begin{array}{rrrrrr}
1 & -1 & -1 & 1 & 0 & 0 \\
0 & 0 & -1 & 1 & 1 & -1 \\
0 & 0 & 1 & -1 & -1 & 1
\end{array}\right)
$$

and

$$
\mathbf{r}(\mathbf{c})=\left(\begin{array}{llllll}
\kappa_{1} c_{1} & \kappa_{2} c_{1}^{2} & \kappa_{3} c_{1} c_{2} & \kappa_{4} c_{3} & \kappa_{5} c_{3} & \kappa_{6} c_{2}
\end{array}\right)^{T}
$$

where $c_{i}$ represents the concentration of $S_{i}, i=1,2,3$.

\section{B. Disturbance modeling}

In this work, disturbances are assumed to act through the change of reaction rate coefficients. For chemical systems, this can be the result of e.g. unexpected change of temperature or chemical composition of a catalyst. In the case of mass convection networks (see e.g. in [20]) which are formally kinetic, change in the 'rate coefficients' can be caused by altered flow conditions. Or, for a kinetic disease model (see e.g. in [21]), changes in the probability of infection or in the healing process might modify the rate coefficients.

The effect of the disturbance on the reaction rate: Consider that in the case of a disturbance event a number of $q \leq$ $r$ elements of the reaction rate coefficient vector $\mathbf{k}$ suffer changes, i.e. a number of $q$ reactions are affected by the disturbance. The rate disturbance vector is defined as $\phi=$ $\left(f_{1} \ldots f_{r}\right)^{T} \in \mathbb{R}^{r}, f_{k} \geq-\kappa_{k} \forall k=1 \ldots r$. The modified rate vector is

$$
\mathbf{k}_{f}=\mathbf{k}+\phi
$$

where $\phi$ may be time-dependent.

If the $k$ th rate is not affected by the disturbance, $f_{k}=0$. If $f_{k}>0$, the reaction rate increases.

If $f_{k}=-\kappa_{k}$, the reaction vanishes.

The CRN model with disturbance reads as

$$
\dot{\mathbf{c}}=N \operatorname{diag}(\mathbf{p}(\mathbf{c}))(\mathbf{k}+\boldsymbol{\phi}) .
$$

Consider the truncated disturbance vector $\mathbf{f}=$ $\left(f_{1} \ldots f_{q}\right)^{T} \in \mathbb{R}^{q}$ containing only those elements of $\phi$ that could take non-zero values in the case of a disturbance event. The corresponding truncated monomial vector is denoted as $\mathbf{p}_{t}\left(\mathbf{c}_{t}\right) \in \mathbb{R}^{q}$. Here $\mathbf{c}_{t}$ represents 
the concentration vector of such species that take part in disturbance-influenced reactions. Let the set of these species be $\mathcal{S}_{t} \subseteq \mathcal{S}$.

The truncated stoichiometric matrix $\left(N_{t} \in R^{n \times q}\right)$ contains those columns of $N$ that describe such reactions that could be influenced by the disturbances. With the appropriately ordered $N_{t}, \mathbf{p}_{t}, \mathbf{f}$, the equation (8) can be rewritten as:

$$
\dot{\mathbf{c}}=N \mathbf{r}(\mathbf{c})+N_{t} P_{t}\left(\mathbf{c}_{t}\right) \mathbf{f}
$$

where $P_{t}\left(\mathbf{c}_{t}\right)=\operatorname{diag}\left(\mathbf{p}_{t}\left(\mathbf{c}_{t}\right)\right)$.

Example 2: (Plain Edelstein Network continued)

Consider that the reactions 1,3 are affected by disturbances. In this case:

$$
\begin{aligned}
& \mathbf{c}_{t}=\left(\begin{array}{ll}
c_{1} & c_{2}
\end{array}\right)^{T}, \quad P_{t}\left(\mathbf{c}_{t}\right)=\operatorname{diag}\left(\begin{array}{ll}
c_{1} & c_{1} c_{2}
\end{array}\right) \\
& N_{t}=\left(\begin{array}{rr}
1 & -1 \\
0 & -1 \\
0 & 1
\end{array}\right) .
\end{aligned}
$$

\section{DISTURBANCE OBSERVER DESIGN FOR CRNS}

This section introduces a diagnosis method to determine rate disturbances in CRNs. The proposed observer-based design is extended to deal with open CRNs, and with partial state measurements.

\section{A. Rate disturbance estimation problem}

Consider the disturbance-affected $\mathrm{CRN}$ model given by the equation (9). The aim of the observer design is to compute an estimate of $\mathbf{f}$ based on which the changes in the dynamics of the CRN can be anticipated.

Let $\Sigma_{\Delta}$ be a dynamic system which has the estimated disturbance vector $\left(\widehat{\mathbf{f}} \in \mathbb{R}^{q}\right.$ ) as output, and its input is $\mathbf{c}_{m} \in$ $\mathbb{R}^{m}$, a vector which contains the measurable entries of the state vector $\mathbf{c}$.

Definition 1: $\Sigma_{\Delta}$ is a disturbance observer of (9) if its internal state vector is bounded and its output satisfies $\widehat{\mathbf{f}} \rightarrow \mathbf{f}$ as $t \rightarrow \infty$ for bounded inputs and finite initial conditions.

If no disturbance is present in the system $(\mathbf{f}=\mathbf{0})$, then $\widehat{\mathbf{f}} \rightarrow \mathbf{0}$. Here $\mathbf{0}=\left(\begin{array}{lll}0 & 0 \ldots 0\end{array}\right)^{T}$.

Relation with the parameter identification problem:

According to the definition of [12], the CRN (3) has uniquely identifiable rate constants assuming that each concentration is measurable, if $N \mathbf{r}\left(\mathbf{c}, \mathbf{k}^{(1)}\right) \neq N \mathbf{r}\left(\mathbf{c}, \mathbf{k}^{(2)}\right) \forall \mathbf{c}$ and $\mathbf{k}^{(1)} \neq \mathbf{k}^{(2)}$. It was shown in [12] that the rate constants are identifiable iff for each reactant complex the vectors of the outgoing reactions are linearly independent.

In the case of the off-line identification, the applied identification methods can be planned in such a way to directly serve the computation of the unknown rate parameters. However, the on-line estimation is based only on instantaneous measurements that cannot be influenced during the observer design. It is why stronger assumptions are necessary to develop an on-line estimation algorithm.

\section{B. Observer design - Full state measurement}

To design a disturbance observer for the system (9), the following assumptions are made:

Assumption 1: The disturbance vector $\mathbf{f}$ is piecewise constant.

Assumption 2: The matrix $N_{t} P_{t}\left(\mathbf{c}_{t}\right)$ has full column rank $\forall \mathbf{c}_{t}$.

If the elements of the vector $c_{t}$ are non-zero during the estimation process, and $N_{t}$ has full column $\operatorname{rank}\left(\operatorname{rank}\left(N_{t}\right)=\right.$ $q$ ) the assumption 2 holds. The full column rank condition is in concordance with the identifiability condition.

Let us construct the observer in the following form

$$
\left\{\begin{array}{l}
\dot{\widehat{\mathbf{c}}}=N \mathbf{r}(\mathbf{c})+N_{t} P_{t}\left(\mathbf{c}_{t}\right) \widehat{\mathbf{f}}+\Gamma_{c}(\mathbf{c}-\widehat{\mathbf{c}}) \\
\dot{\hat{\mathbf{f}}}=P_{t}\left(\mathbf{c}_{t}\right) N_{t}^{T} \Gamma_{f}(\mathbf{c}-\widehat{\mathbf{c}})
\end{array}\right.
$$

where $\Gamma_{f} \in \mathbb{R}^{n \times n}$ is a diagonal, positive definite matrix, $\Gamma_{c} \in \mathbb{R}^{n \times n}$ is a positive definite symmetric matrix. The estimated disturbance is denoted by $\widehat{\mathbf{f}}$, the estimated state vector is $\widehat{\mathbf{c}}$. The output of (12) is $\widehat{\mathbf{f}}$.

Theorem 1: If the Assumptions 1, 2 hold, then (12) is a disturbance observer of the system (9).

Proof: Based on the models (9), (12) and Assumption 1 the dynamics of the observation errors $(\widetilde{\mathbf{c}}=\mathbf{c}-\widehat{\mathbf{c}}, \widetilde{\mathbf{f}}=\mathbf{f}-\widehat{\mathbf{f}})$ yields as

$$
\left(\begin{array}{c}
\dot{\tilde{\mathbf{c}}} \\
\dot{\tilde{\mathbf{f}}}
\end{array}\right)=\left(\begin{array}{cc}
-\Gamma_{c} & N_{t} P_{t}\left(\mathbf{c}_{t}\right) \\
-P_{t}\left(\mathbf{c}_{t}\right) N_{t}^{T} \Gamma_{f} & 0
\end{array}\right)\left(\begin{array}{c}
\widetilde{\mathbf{c}} \\
\widetilde{\mathbf{f}}
\end{array}\right) .
$$

Define the Lyapunov function candidate

$$
L(t)=\frac{1}{2} \widetilde{\mathbf{c}}^{T} \Gamma_{f} \widetilde{\mathbf{c}}+\frac{1}{2} \widetilde{\mathbf{f}}^{T} \widetilde{\mathbf{f}} .
$$

The time-derivative of it reads as

$$
\dot{L}(t)=\widetilde{\mathbf{c}}^{T} \Gamma_{f} \dot{\widetilde{\mathbf{c}}}+\widetilde{\mathbf{f}}^{T} \dot{\tilde{\mathbf{f}}}
$$

From the model (13) we obtain:

$$
\dot{L}(t)=\widetilde{\mathbf{c}}^{T} \Gamma_{f}\left(-\Gamma_{c} \widetilde{\mathbf{c}}+N_{t} P_{t}\left(\mathbf{c}_{t}\right) \widetilde{\mathbf{f}}\right)-\widetilde{\mathbf{f}}^{T} P_{t}\left(\mathbf{c}_{t}\right) N_{t}^{T} \Gamma_{f} \widetilde{\mathbf{c}} .
$$

As $\widetilde{\mathbf{c}}^{T} \Gamma_{f} N_{t} P_{t}\left(\mathbf{c}_{t}\right) \widetilde{\mathbf{f}}=\left(\widetilde{\mathbf{c}}^{T} \Gamma_{f} N_{t} P_{t}\left(\mathbf{c}_{t}\right) \widetilde{\mathbf{f}}\right)^{T}$ (it is scalar), it yields:

$$
\widetilde{\mathbf{c}}^{T} \Gamma_{f} N_{t} P_{t}\left(\mathbf{c}_{t}\right) \widetilde{\mathbf{f}}=\widetilde{\mathbf{f}}^{T} P_{t}\left(\mathbf{c}_{t}\right) N_{t}^{T} \Gamma_{f} \widetilde{\mathbf{c}}
$$

Consequently, taking into account that $\Gamma_{f} \Gamma_{c}$ is positive definite:

$$
\dot{L}(t)=-\widetilde{\mathbf{c}}^{T} \Gamma_{f} \Gamma_{c} \widetilde{\mathbf{c}} \leq 0
$$

By LaSalle invariance principle, the trajectories of the system (13) converge to the invariant set $\widetilde{\mathbf{c}}=\mathbf{0}$. Accordingly, $\widetilde{\mathbf{c}}, \dot{\widetilde{\mathbf{c}}} \rightarrow \mathbf{0}$ as $t \rightarrow \infty$. Hence, by (13) we obtain that $N_{t} P_{t}\left(\mathbf{c}_{t}\right) \widetilde{\mathbf{f}} \rightarrow \mathbf{0}$ as $t \rightarrow \infty$. By Assumption 2 the matrix $N_{t} P_{t}\left(\mathbf{c}_{t}\right)$ admits left inverse. Then we conclude that $\widehat{\mathbf{f}} \rightarrow \mathbf{f}$ as $t \rightarrow \infty$. 


\section{Extended observer with input disturbance compensation}

Consider the model of an open CRN, with input and output flows as in [22]

$$
\dot{\mathbf{c}}=N \mathbf{r}(\mathbf{c})+\mathbf{i}-V_{o} \mathbf{c} .
$$

The vector of the rate of supply is $\mathbf{i}, V_{o} \mathbf{c}$ is the rate of removal. $V_{o}=\operatorname{diag}(v v \ldots v)$ where $v \geq 0$ is the outflow rate.

The rate of supply can be modelled as

$$
\mathbf{i}=\left(\begin{array}{llll}
v_{I 1} c_{I 1} & v_{I 2} c_{I 2} & \ldots & v_{I n} c_{I n}
\end{array}\right)^{T}
$$

where $c_{I i}$ is the $i$ th inlet concentration and $v_{I i} \geq 0$ is the $i$ th input flow rate.

If constant volume is assumed in the reactor where the reaction takes place, the relation $\sum_{i=1}^{n} v_{I i}=v$ holds.

The input disturbance is considered as a change in the inlet concentration and it is modeled as an additive term in the open CRN model in the form $E \mathbf{d}$, where $\mathbf{d} \in \mathbb{R}^{p}$ is the input disturbance vector and $E \in \mathbb{R}^{n \times p}$ is the input disturbance matrix, containing $p$ standard basis vectors with dimension $n$, indicating that which species' concentrations are influenced by the disturbance.

Assumption 3: The input disturbance vector $\mathbf{d}$ is piecewise constant.

By (9), the open CRN model with rate- and input disturbance has the form:

$$
\dot{\mathbf{c}}=N \mathbf{r}(\mathbf{c})+N_{t} P_{t}\left(\mathbf{c}_{t}\right) \mathbf{f}+\mathbf{i}+E \mathbf{d}-V_{o} \mathbf{c} .
$$

Formulate the augmented observer as

$$
\left\{\begin{array}{l}
\dot{\hat{\mathbf{c}}}=N \mathbf{r}(\mathbf{c})+N_{t} P_{t}\left(\mathbf{c}_{t}\right) \widehat{\mathbf{f}}+\mathbf{i}-V_{o} \mathbf{c}+E \widehat{\mathbf{d}}+\Gamma_{c}(\mathbf{c}-\widehat{\mathbf{c}}) \\
\dot{\hat{\mathbf{f}}}=P_{t}\left(\mathbf{c}_{t}\right) N_{t}^{T} \Gamma_{f}(\mathbf{c}-\widehat{\mathbf{c}}) \\
\dot{\hat{\mathbf{d}}}=E^{T} \Gamma_{f}(\mathbf{c}-\widehat{\mathbf{c}}) .
\end{array}\right.
$$

Assumption 4: The state dependent matrix $N_{E}\left(\mathbf{c}_{t}\right)=$ $\left[N_{t} P_{t}\left(\mathbf{c}_{t}\right) E\right]$ has full column rank $\forall \mathbf{c}_{t}$.

Note that for Assumption 4 it is necessary that $\operatorname{dim}(\mathbf{f})+$ $\operatorname{dim}(\mathbf{d}) \leq n$.

Corollary 1: If the Assumptions 1, 3, 4 hold, then (22) is a disturbance observer of the system (21). Moreover, $\lim _{t \rightarrow \infty} \widehat{\mathbf{d}}=\mathbf{d}$.

This corollary can be proven applying similar considerations as in the proof of Theorem 1, by using the Lyapunov function candidate

$$
L_{d}(t)=\frac{1}{2} \widetilde{\mathbf{c}}^{T} \Gamma_{f} \widetilde{\mathbf{c}}+\frac{1}{2} \widetilde{\mathbf{f}}^{T} \widetilde{\mathbf{f}}+\frac{1}{2} \widetilde{\mathbf{d}} \widetilde{\mathbf{d}}^{T}
$$

In this case, the disturbance observer's output vector is $\left(\widetilde{\mathbf{f}}^{T} \widetilde{\mathbf{d}}^{T}\right)^{T}$.

Example 3: (Plain Edelstein Network continued)

Consider two cases for the input disturbance matrices:

$$
E_{1}=\left(\begin{array}{lll}
1 & 0 & 0
\end{array}\right)^{T} \text { and } E_{2}=\left(\begin{array}{lll}
0 & 1 & 0
\end{array}\right)^{T} \text {. }
$$

For these two cases, by (11), the state dependent matrices $N_{E}$, defined in Assumption 4, take the forms:

$$
N_{E 1}=\left(\begin{array}{rrr}
c_{1} & -c_{1} c_{2} & 1 \\
0 & -c_{1} c_{2} & 0 \\
0 & c_{1} c_{2} & 0
\end{array}\right), N_{E 2}=\left(\begin{array}{rrr}
c_{1} & -c_{1} c_{2} & 0 \\
0 & -c_{1} c_{2} & 1 \\
0 & c_{1} c_{2} & 0
\end{array}\right) \text {. }
$$

It can be seen that $\operatorname{rank}\left(N_{E 1}\right)=2, \operatorname{rank}\left(N_{E 2}\right)=3$ $\forall c_{1}, c_{2}>0$. Hence, the disturbance estimation problem is solvable for the disturbance input matrix $E_{2}$.

\section{Disturbance observer in the partial state measurement} case

Partition the state vector of the CRN as

$$
\mathbf{c}=\left(\mathbf{c}_{m}^{T} \mathbf{c}_{u}^{T}\right)^{T}
$$

where the entries of $\mathbf{c}_{m} \in \mathbb{R}_{+}^{m}$ are measurable $(m \leq n)$. The concentrations in the vector $\mathbf{c}_{u} \in \mathbb{R}_{+}^{n-m}$ are not available for the estimation.

The vector of monomials, introduced in (2), is also partitioned as:

$$
\boldsymbol{p}(\mathbf{c})=\left(\mathbf{p}_{m}\left(\mathbf{c}_{m}\right)^{T} \mathbf{p}_{u}(\mathbf{c})^{T}\right)^{T}
$$

where $\mathbf{p}_{m}\left(\mathbf{c}_{m}\right) \in \mathbb{R}_{+}^{\mu}$ contains those monomials which depend only on $\mathbf{c}_{m}(0<\mu \leq r)$.

Based on the model (3), the dynamics of $\mathbf{c}_{m}$ reads as:

$$
\dot{\mathbf{c}}_{m}=N_{m} P_{m}\left(\mathbf{c}_{m}\right) \mathbf{k}_{m}+N_{u} K_{u} \mathbf{p}_{u}(\mathbf{c})
$$

Here $P_{m}\left(\mathbf{c}_{m}\right)=\operatorname{diag}\left(\mathbf{p}_{m}\left(\mathbf{c}_{m}\right)\right), N_{m}$ contains those entries of the stoichiometric matrix the row index of which correspond to a measurable state and the column index coincide with the known monomial vector terms. $\mathbf{k}_{m} \in \mathbb{R}_{+}^{\mu}$ are the rate coefficients that correspond to the known monomial vector terms, $K_{u}=\operatorname{diag}\left(\mathbf{k}_{u}\right)$.

Similar to the disturbance modeling approach presented in (7) and (8), consider that $\mathbf{k}_{m}$ is influenced by an unknown (22)additive disturbance term in the form $\mathbf{k}_{m f}=\mathbf{k}_{m}+\mathbf{f}$.

Assumption 5: The matrix $N_{m} P_{m}\left(\mathbf{c}_{m}\right)$ has full column rank $\forall \mathbf{c}_{m}$.

Assumption 6: The elements of the vector $\mathbf{p}_{u}(\mathbf{c})$ are vanishing, i.e. $\exists \mathbf{w}(t) \in \mathbb{R}_{+}^{\mu}$ such that $\lim _{t \rightarrow \infty} \mathbf{w}(t)=\mathbf{0}$, and $\left|N_{m} K_{m} \mathbf{p}_{u}(\mathbf{c}(t))\right| \leq \mathbf{w}(t) \forall t \geq 0$ element-vise.

Example 4: Let the reaction network be the following:

$$
\begin{array}{r}
A+B \stackrel{\kappa_{1}}{\underset{\kappa_{2}}{\rightleftharpoons}} C \\
C \stackrel{\kappa_{3}}{\longrightarrow} D \\
3 B \stackrel{\kappa_{4}}{\longrightarrow} E
\end{array}
$$

The stoichiometric matrix of it reads as:

$$
N=\left(\begin{array}{rrrr}
-1 & 1 & 0 & 0 \\
-1 & 1 & 0 & -1 \\
1 & -1 & -1 & 0 \\
0 & 0 & 1 & 0 \\
0 & 0 & 0 & 3
\end{array}\right)
$$

and

$$
\begin{gathered}
\mathbf{c}=\left(\begin{array}{lllll}
c_{A} & c_{B} & c_{C} & c_{D} & c_{E}
\end{array}\right)^{T} \\
\mathbf{r}(\mathbf{c})=\left(\begin{array}{llll}
\kappa_{1} c_{A} c_{B} & \kappa_{2} c_{C} & \kappa_{3} c_{C} & \kappa_{4} c_{B}^{3}
\end{array}\right)^{T}
\end{gathered}
$$

It can be seen that the concentration state $c_{C}$ is vanishing. 
Consider that the concentrations of the reactants $c_{A}$ and $c_{B}$ are measurable. The dynamics of the measurable states reads as:

$$
\begin{array}{r}
\left(\begin{array}{c}
\dot{c}_{A} \\
\dot{c}_{B}
\end{array}\right)=\left(\begin{array}{rr}
-1 & 0 \\
-1 & -1
\end{array}\right)\left(\begin{array}{rr}
c_{A} c_{B} & 0 \\
0 & c_{B}^{3}
\end{array}\right)\left(\begin{array}{c}
\kappa_{1} \\
\kappa_{4}
\end{array}\right)+ \\
\left(\begin{array}{c}
-1 \\
-1
\end{array}\right) \kappa_{2} c_{C}
\end{array}
$$

Let the disturbance observer algorithm be given in the following form:

$$
\left\{\begin{array}{c}
\dot{\hat{\mathbf{c}}}_{m}=N_{m} P_{m}\left(\mathbf{c}_{m}\right)\left(\mathbf{k}_{m}+\widehat{\mathbf{f}}\right)+\Gamma_{c}\left(\mathbf{c}_{m}-\widehat{\mathbf{c}}_{m}\right) \\
\quad+W \operatorname{sgn}\left(\mathbf{c}_{m}-\widehat{\mathbf{c}}_{m}\right) \\
\dot{\hat{\mathbf{f}}}=P_{m}\left(\mathbf{c}_{m}\right) N_{m}^{T} \Gamma\left(\mathbf{c}_{m}-\widehat{\mathbf{c}}_{m}\right)
\end{array}\right.
$$

Here $W=\operatorname{diag}(\mathbf{w}), \Gamma_{c} \in \mathbb{R}^{\mu \times \mu}$ is a positive definite symmetric matrix, $\Gamma \in \mathbb{R}^{\mu \times \mu}$ is a positive definite diagonal matrix, and the sign function $\operatorname{sgn}(\cdot)$ applies element-wise to the vector.

Theorem 2: If the Assumptions 1, 5 and 6 hold, then (32) is a disturbance observer of the system (27).

Proof: Based on the equations (27) and (32) the dynamics of the observation errors $\left(\widetilde{\mathbf{c}}_{m}=\mathbf{c}_{m}-\widehat{\mathbf{c}}_{m}, \widetilde{\mathbf{f}}=\mathbf{f}-\widehat{\mathbf{f}}\right)$ yields as:

$$
\begin{gathered}
\left(\begin{array}{c}
\dot{\widetilde{\mathbf{c}}}_{m} \\
\dot{\tilde{\mathbf{f}}}_{m}
\end{array}\right)=\left(\begin{array}{cc}
-\Gamma_{c} & N_{m} P_{m}\left(\mathbf{c}_{m}\right) \\
-P_{m}\left(\mathbf{c}_{m}\right) N_{m}^{T} \Gamma & 0
\end{array}\right)\left(\begin{array}{c}
\widetilde{\mathbf{c}}_{m} \\
\widetilde{\mathbf{f}}
\end{array}\right) \\
+\left(\begin{array}{c}
N_{u} K_{u} \mathbf{p}_{u}(\mathbf{c})-W \operatorname{sgn}\left(\widetilde{\mathbf{c}}_{m}\right) \\
0
\end{array}\right) .
\end{gathered}
$$

The Lyapunov function candidate for the convergence analysis is chosen as

$$
L_{m}(t)=\frac{1}{2} \widetilde{\mathbf{c}}_{m}^{T} \Gamma \widetilde{\mathbf{c}}_{m}+\frac{1}{2} \widetilde{\mathbf{f}}^{T} \widetilde{\mathbf{f}}
$$

The time-derivative of it reads as, see the model (33):

$$
\begin{aligned}
\dot{L}_{m}(t) & =\widetilde{\mathbf{c}}^{T} \Gamma\left(-\Gamma_{c} \widetilde{\mathbf{c}}_{m}+N_{m} P_{m}\left(\mathbf{c}_{m}\right) \widetilde{\mathbf{f}}\right) \\
& -\widetilde{\mathbf{f}}^{T} P_{m}\left(\mathbf{c}_{m}\right) N_{m}^{T} \Gamma \widetilde{\mathbf{c}}_{m} \\
& +\widetilde{\mathbf{c}}^{T}\left(N_{u} K_{u} \mathbf{p}_{u}(\mathbf{c})-W \operatorname{sgn}\left(\widetilde{\mathbf{c}}_{m}\right)\right) .
\end{aligned}
$$

By Assumption 6 yields that $\widetilde{\mathbf{c}}^{T} N_{u} K_{u} \mathbf{p}_{u}(\mathbf{c}) \leq|\widetilde{\mathbf{c}}| W$. Consequently, by taking into account that $\Gamma_{f} \Gamma_{c}$ is symmetric positive definite, it results:

$$
\dot{L}_{m}(t) \leq-\widetilde{\mathbf{c}}_{m}^{T} \Gamma \Gamma_{c} \widetilde{\mathbf{c}}_{m} \leq 0 .
$$

Accordingly, the Lyapunov function (34) is nonincreasing, hence $\widetilde{\mathbf{c}}_{m i}, \widetilde{\mathbf{f}}_{i}$ are bounded vectors, i.e. $\widetilde{\mathbf{c}}_{m i}$, $\widetilde{\mathbf{f}}_{i} \in \mathcal{L}_{\infty}$ for each entry $i$.

By Assumption 6 and the observer error dynamics (33), it also yields that $\dot{\widetilde{\mathbf{c}}}_{m i} \in \mathcal{L}_{\infty}, \forall i$.

The relation (36) can be reformulated as $\int_{0}^{\infty} \widetilde{\mathbf{c}}_{m}^{T} \Gamma \Gamma_{c} \widetilde{\mathbf{c}}_{m} d \tau \leq L_{m}(0)-L_{m \infty}$ where $L_{m \infty}=$ $\lim _{t \rightarrow \infty} L_{m}(t)$. It yields that $\mathbf{c}_{m i} \in \mathcal{L}_{2}, \forall i$.

As $\widetilde{\mathbf{c}}_{m i} \in \mathcal{L}_{\infty}, \dot{\widetilde{\mathbf{c}}}_{m i} \in \mathcal{L}_{\infty}, \widetilde{\mathbf{c}}_{m i} \in \mathcal{L}_{2}$, it results that $\lim _{t \rightarrow \infty} \widetilde{\mathbf{c}}_{m i}=0, \forall i$.

By Assumptions 5, 6 and the observer error dynamics (33) it also yields that $\lim _{t \rightarrow \infty} \widetilde{\mathbf{f}}_{i}=0, \forall i$.

\section{Simulation CASE STUdies}

Two simulation experiments were performed in Matlab/ Simulink environment to examine the performance of the proposed disturbance estimation method.

\section{A. E1: Disturbance estimation - full state measurement}

The observer proposed in subsection III-B was tested on an Edelstein network, that was introduced in Examples 1-3. The dynamic model is given by the relations (3), (5), (6). The reaction rate coefficients were chosen $\kappa_{k}=1, k=1 \ldots 6$.

For the first experiment (E1) in the Edelstein network the following reaction rate changes were assumed: $\kappa_{f 1}=\kappa_{1}+$ $f_{1}, \kappa_{f 3}=\kappa_{3}+f_{3}$ where $f_{1}=0.1 \cdot \mathbf{1}(t-25)$ and $f_{3}=$ $-0.2 \cdot \mathbf{1}(t-50)$, where $\mathbf{1}(\cdot)$ denotes the unit step function.

The observer (12) for the Edelstein network was implemented with the following gain matrices: $\Gamma_{c}=$ $\operatorname{diag}\left(\begin{array}{lll}1 & 1 & 0.75\end{array}\right), \Gamma_{f}=\operatorname{diag}\left(\begin{array}{lll}1 & 1 & 1\end{array}\right)$. The matrices $P_{t}$ and $N_{t}$ are given by the equations (10) and (11) respectively.

The evolution of the CRN states and the estimated disturbance signals are presented in Figures 1 and 2 .

\section{B. E2: Disturbance estimation - partial state measurement}

During the second experiment (E2) the CRN presented in

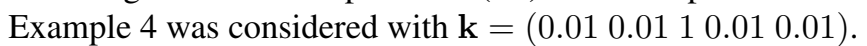
The disturbances were chosen as: $f_{1}=-0.005 \cdot \mathbf{1}(t-10)$, ${ }^{3} f_{4}=0.005 \cdot \mathbf{1}(t-30)$.

The measurable states were $c_{A}$ and $c_{B}$, the augmented observer (32) was designed based on the model (31) with the parameters $\Gamma_{c}=\operatorname{diag}(2020), \Gamma_{f}=\operatorname{diag}(2020)$, and $\mathbf{w}_{1}=\mathbf{w}_{2}=\exp (-10 t)$.

For this simulation experiment the estimated and real CRN state trajectories and the estimated disturbance signals are presented in Figures 3 and 4.

In both cases ( $E 1$ and $E 2)$ the experimental measurements show the convergence of the estimated rate disturbance signals to their real values.

\section{CONCLuSions}

Based on the ODE model of mass action CRNs, nonlinear observers were proposed that are able to estimate on-line disturbances in reaction rate coefficients using the measured concentrations as state variables. Based on the algebraic structure of kinetic models, the convergence of the estimated disturbance vector to the real one was proven using a suitable Lyapunov function candidate. The method was extended to simultaneously detect disturbances in the input of open CRNs

A second extension was also proposed for the case when only partial state measurements are available. For this case additional assumptions on some of the unmeasurable states are necessary, and the problem is solvable using robust estimation algorithm.

Simulation measurements show that the proposed observers can precisely estimate the disturbance induced changes in the reaction rate coefficients. 

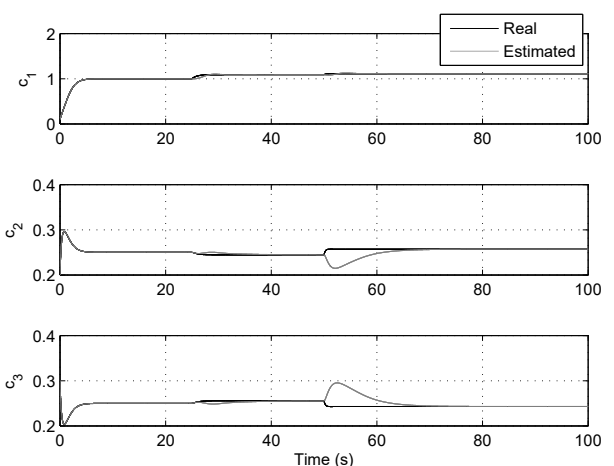

Fig. 1. CRN states with two rate disturbances (Case E1)
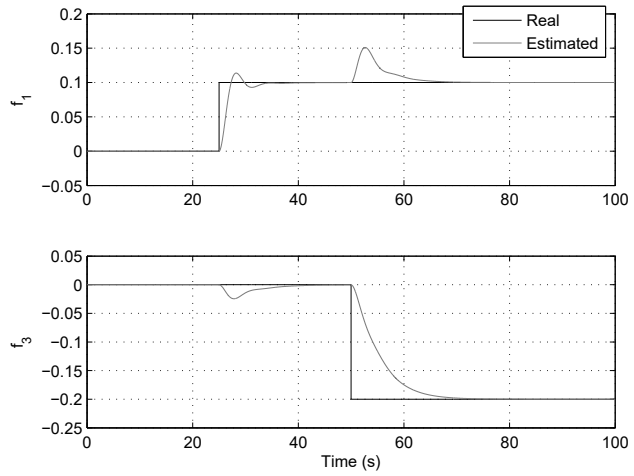

Fig. 2. Estimation with two rate disturbances (Case E1)

\section{REFERENCES}

[1] G. Besanon, Nonlinear Observers and Applications. Springer-Verlag Berlin Heidelberg, 2007.

[2] P. Érdi and J. Tóth, Mathematical Models of Chemical Reactions. Theory and Applications of Deterministic and Stochastic Models. Manchester, Princeton: Manchester University Press, Princeton University Press, 1989.

[3] K. Hangos and I. Cameron, Process modelling and model analysis. Academic Press, London, 2001.

[4] D. F. Anderson, "Tutorial: chemical reaction network theory for both deterministic and stochastic models," in Programming with Chemical Reaction Networks: Mathematical Foundations, 2014.

[5] M. Feinberg, Lectures on chemical reaction networks. Notes of lectures given at the Mathematics Research Center, University of Wisconsin, 1979.

[6] D. Angeli, "A tutorial on chemical network dynamics," European Journal of Control, vol. 15, pp. 398-406, 2009.

[7] M. Chaves and E. D. Sontag, "State-estimators for chemical reaction networks of Feinberg-Horn-Jackson zero deficiency type," European Journal of Control, vol. 8, no. 4, pp. 343 - 359, 2002.

[8] G. Lipták, G. Szederkényi, and M. Hangos, "Kinetic feedback design for polynomial systems," Journal of Process Control, vol. 41, pp. 5666, 2016.

[9] M. Farina and S. Bittanti, "An observer for mass-action chemical reaction networks," European Journal of Control, vol. 15, no. 5, pp. $578-593,2009$.

[10] S. Burnham, M. Willis, and A. Wright, "Identifying chemical reaction network models," 8th IFAC Symposium on Dynamics and Control of Process System, vol. 40, no. 5, pp. 225 - 230, 2007.

[11] M. Brendel, D. Bonvin, and W. Marquardt, "Incremental identification of kinetic models for homogeneous reaction systems," Chemical Engineering Science, vol. 61, no. 16, pp. 5404 - 5420, 2006.

[12] G. Craciun and C. Pantea, "Identifiability of chemical reaction networks," Journal of Mathematical Chemistry, vol. 44, no. 1, pp. 244 259,2008
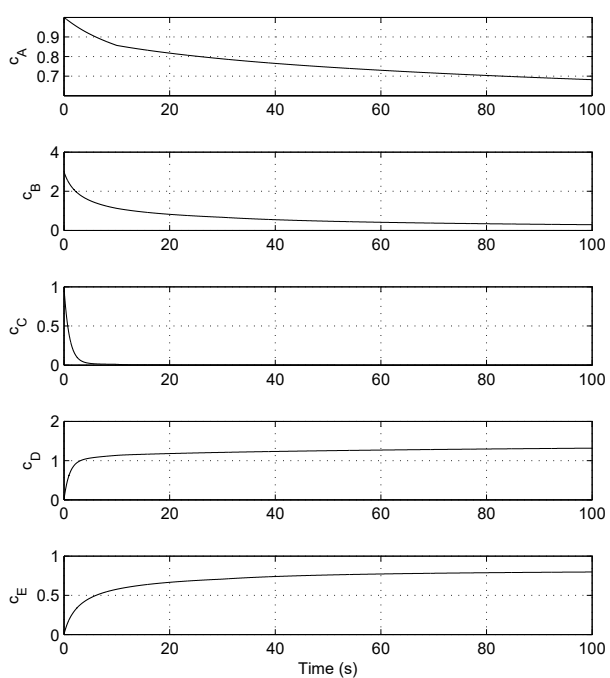

Fig. 3. CRN states with two rate disturbances (Case E2)
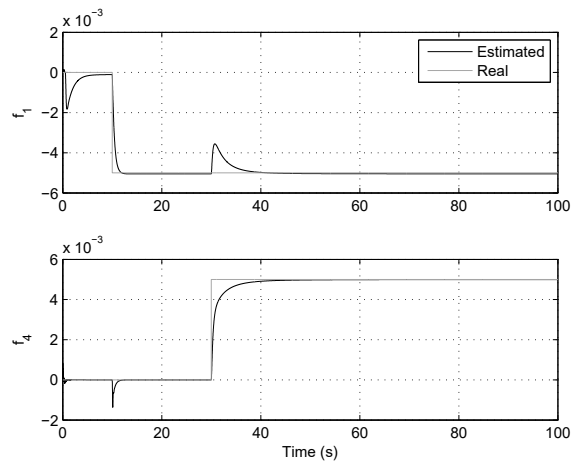

Fig. 4. Estimation with two rate disturbances (Case E2)

[13] O.-T. Chis, J. R. Banga, and E. Balsa-Canto, "Structural identifiability of systems biology models: A critical comparison of methods," PLOS ONE, vol. 6, no. 11, pp. 1-16, 112011.

[14] J. M. Ali, N. H. Hoang, M. Hussain, and D. Dochain, "Review and classification of recent observers applied in chemical process systems," Computers \& Chemical Engineering, vol. 76, no. Supplement C, pp. $27-41,2015$.

[15] J. Valluru, S. C. Patwardhan, and L. T. Biegler, "Development of robust extended Kalman filter and moving window estimator for simultaneous state and parameter/disturbance estimation," Journal of Process Control, vol. 69, pp. 158 - 178, 2018.

[16] M. Kano, S. Lee, and S. Hasebe, "Two-stage subspace identification for softsensor design and disturbance estimation," Journal of Process Control, vol. 19, no. 2, pp. 179 - 186, 2009.

[17] F. Horn and R. Jackson, "General mass action kinetics," Archive for Rational Mechanics and Analysis, vol. 47, pp. 81-116, 1972.

[18] B. B. Edelstein, "Biochemical model with multiple steady states and hysteresis," Journal of Theoretical Biology, vol. 29, pp. 57-62, 1970.

[19] I. Martinez-Forero, A. Peláez-López, and P. Villoslada, "Steady state detection of chemical reaction networks using a simplified analytical method," PLOS ONE, vol. 5, no. 6, pp. 1-6, 2010.

[20] M. Cantoni, E. Weyer, Y. Li, S. K. Ooi, I. Mareels, and M. Ryan, "Control of large-scale irrigation networks," Proceedings of the IEEE, vol. 95, no. 1, pp. 75-91, 2007.

[21] J. M. Epstein, J. Parker, D. Cummings, and R. A. Hammond, "Coupled contagion dynamics of fear and disease: mathematical and computational explorations," PLOS ONE, vol. 3, no. 12, p. e3955, 2008.

[22] G. Bastin and J. V. Impe, "Nonlinear and adaptive control in biotechnology: A tutorial," European Journal of Control, vol. 1, no. 1, pp. $37-53,1995$. 